\title{
Fracture behaviors of thin superconducting films with field-dependent critical current density
}

\author{
An He, Cun Xue, Huadong Yong, Youhe Zhou*
}

Department of Mechanics and Engineering Sciences, School of Civil Engineering and Mechanics, Lanzhou University, Lanzhou, Gansu 730000, P.R. China

Key Laboratory of Mechanics on Disaster and Environment in Western China attached to the Ministry of Education of China

*Corresponding author: zhouyh@lzu.edu.cn

\begin{abstract}
The fracture behaviors under electromagnetic force with field-dependent critical current density in thin superconducting film are investigated. Applying finite element method, the stress intensity factors of one central crack versus applied field and crack length are obtained for the exponential model, which was compared with the Bean model and Kim model. This work can offer good estimations and provide a basis for interpretation of cracking and mechanical failure of HTS thin films in numerous real situations.
\end{abstract}

Keywords Fracture behaviors, Superconducting film, Stress intensity factor, Exponential model

\section{Introduction}

High-temperature superconductor (HTS) tapes have provided a variety of engineering applications, such as ac power transmission cables [1, 2], filters for wireless communication [3]. With the development of engineering applications, major efforts are directed towards improvements on mechanical performances of HTS bulks in the presence of high magnetic field. During the past years, a series of investigations were devoted to the magneto-elastic behaviors induced by flux-pinning in bulk superconductors for different sample shapes, critical state models and magnetization processes [4-6]. For example, Johansen studied magnetostrictive behavior during the field activation processes, and revealed that the giant irreversible magnetostriction and internal stress could often lead to fatal cracking of the HTS bulks [7]. It is well established experimentally that when an HTS bulk traps large magnetic fields, the mechanical stability can be threatened by the tensile stress induced by flux-pinning [8]. Due to their low tensile strength of melt-processed HTS materials, micro-cracks and macro-cracks parallel to the $a-b$ plane were both observed in large grain bulk $\mathrm{RE}-\mathrm{Ba}-\mathrm{Cu}-\mathrm{O}$ (RE: rare earth elements) superconductors [9, 10]. In recent years, many theoretical analyses for the crack problem of long rectangular slabs and long cylinders under electromagnetic force have been achieved [11-13]. Very recently, the central crack and interface crack problem of a thin superconducting strip were investigated by a field-independent $J_{c}$ (the Bean model) $[14,15]$.

However, it is observed experimentally that the critical current density strongly depends on the flux density in real materials. The body forces induced by flux-pinning and magneto-elastic behavior are significantly different in magnitude between the Kim model and Bean model in thin superconducting strip [16]. It is found that Kim model is in much better agreement with experiment than Bean model at low temperatures. Furthermore, the exponential model, on the other hand, is one of the models that has been recently discussed in relation to the high-temperature superconductors [17-19]. Therefore, it is very worthwhile to investigate crack problem of HTS thin films for the exponential model. Since the superconducting materials are brittle, the stress intensity factor is a good fracture criterion and can provide successful prediction for fracture behavior. Our main purpose of this paper is to study the fracture behaviors in a HTS thin film with one central 
longitudinal crack under electromagnetic force. Compared with the Bean model and Kim model, we investigate center-situated crack problem in an infinitely long superconducting film for the exponential model. The stress intensity factors at the crack tips are obtained by means of finite element method, which are discussed for different applied fields and crack lengths. In our calculations, the Young's modulus and the Poisson's ratio are assumed to be $125 \mathrm{GPa}$ and 0.3 as adopted in Ref. [17].

\section{Basic equations}

In the present article, we consider a thin film with one central crack in a perpendicular applied magnetic field, and the film is located in the Cartesian coordinate system [see Fig. 1]. The width and the thickness of the film is $2 w$ and $d$, respectively. The film is assumed to be isotropic and infinite along the $x$ axis. Due to the symmetry of the problem, the current density has only a $x$ component $J_{x}(y)$. Since the crack is parallel to the current, the disturbance of crack on the current and flux are negligible. The current density $J_{x}(y)$ and flux density $B_{z}(y)$ can be regarded as average value over the film thickness in case of $d<<w$. Based on the theory of field-dependent current [20], for the applied magnetic field increasing from zero to $B_{a m}$, the profiles of current and flux density can be calculated numerically from the equations below

$$
\begin{aligned}
& J_{x}(y)=\left\{\begin{array}{l}
-\frac{2}{\pi} y \sqrt{b^{2}-y^{2}} \int_{b}^{w} \frac{J_{c}\left(B_{z}\left(y^{\prime}\right)\right) d y^{\prime}}{\left(y^{\prime 2}-y^{2}\right) \sqrt{y^{\prime 2}-b^{2}}},|y|<b \\
-\frac{y}{|y|} J_{c}\left(B_{z}(y)\right), \\
B_{z}(y)=B_{f}|y| \sqrt{y^{2}-b^{2}} \int_{b}^{w} \frac{J_{c}\left(B_{z}\left(y^{\prime}\right)\right)}{J_{c 0}} \frac{d y^{\prime}}{\left(y^{2}-y^{\prime 2}\right) \sqrt{y^{\prime 2}-b^{2}}}, b<|y| \neq w,
\end{array}\right. \\
& B_{a}=B_{f} \int_{b}^{w} \frac{J_{c}\left(B_{z}\left(y^{\prime}\right)\right)}{J_{c 0}} \frac{d y^{\prime}}{\sqrt{y^{\prime 2}-b^{2}}},
\end{aligned}
$$

and the scaling field $B_{f}$ is defined by

$$
B_{f}=\frac{\mu_{0} J_{c 0} d}{\pi},
$$

where $J_{c 0}$ and $\mu_{0}$ is the zero-field critical current and the permeability of free space, respectively.

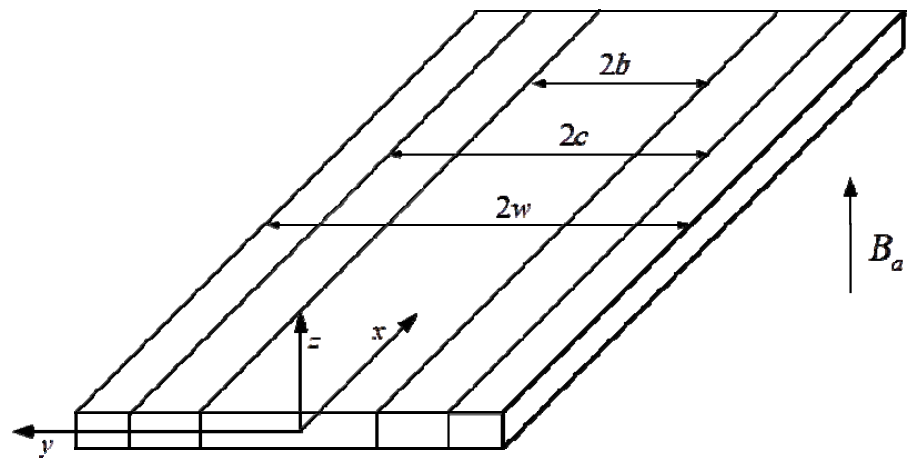

(a) 


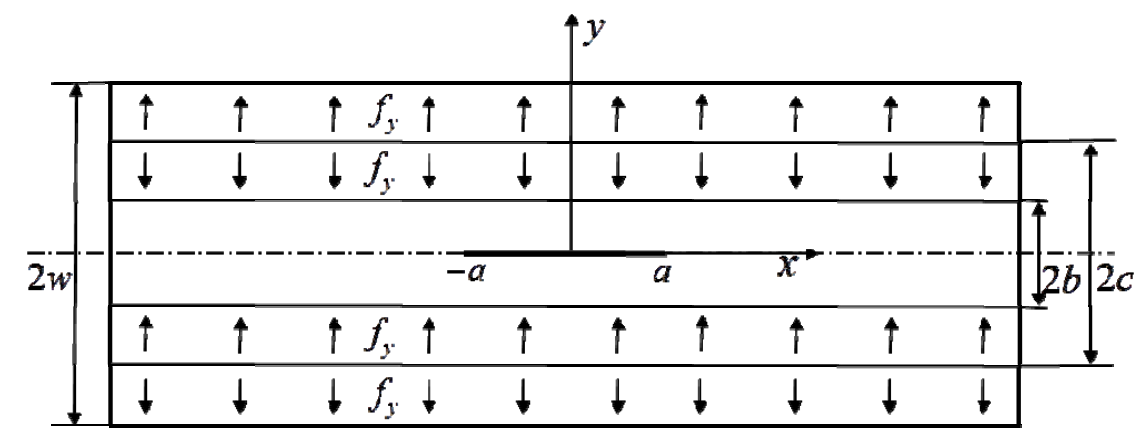

(b)

Figure 1. (a) Schematic of the superconducting thin film and coordinate system in a perpendicular applied field. The superconducting film subjected to the Lorentz force with one central crack.

When the applied magnetic field decreases from initial value $B_{a m}$ to $B_{a}$, the flux density changes in the outer region $(c<|x|<w)$ but is unchanged in the inner region $(b<|x|<c)$, and the central region $(|x|<b)$ remains free of flux. Therefore, the distributions of current and flux density can be obtained by solving the following equations

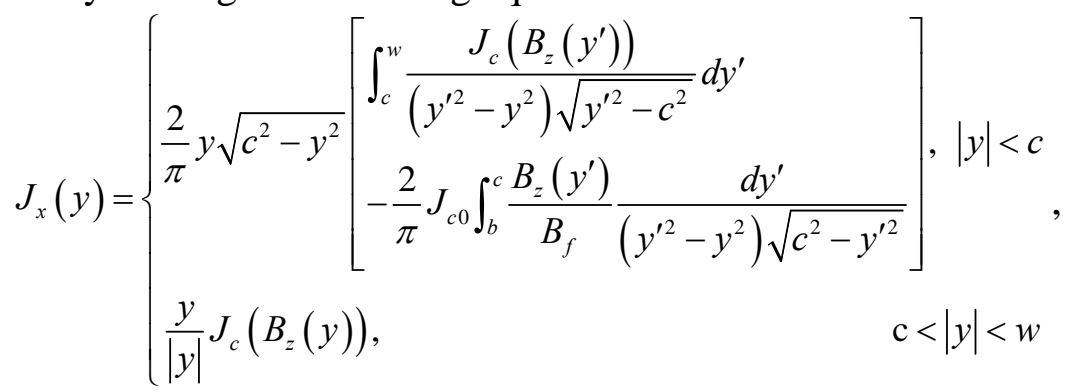

$$
\begin{aligned}
& B_{z}(y)=|y| \sqrt{y^{2}-c^{2}}\left[\begin{array}{l}
-B_{f} \int_{c}^{w} \frac{J_{c}\left(B_{z}\left(y^{\prime}\right)\right)}{J_{c 0}} \frac{d y^{\prime}}{\left(y^{2}-y^{\prime 2}\right) \sqrt{y^{\prime 2}-c^{2}}} \\
+\frac{2}{\pi} \int_{b}^{c} \frac{B_{z}\left(y^{\prime}\right)}{\left(y^{2}-y^{\prime 2}\right) \sqrt{c^{2}-y^{\prime 2}}} d y^{\prime}
\end{array}\right], \mathrm{c}<|y| \neq w, \\
& B_{a}=-B_{f} \int_{c}^{w} \frac{J_{c}\left(B_{z}\left(y^{\prime}\right)\right)}{J_{c 0}} \frac{d y^{\prime}}{\sqrt{y^{\prime 2}-c^{2}}}+\frac{2}{\pi} \int_{b}^{c} \frac{B_{z}\left(y^{\prime}\right)}{\sqrt{c^{2}-y^{\prime 2}}} d y^{\prime} \text {. }
\end{aligned}
$$

In the Bean model, the critical current density is constant in the film. While in reality the critical current density is dependent on the flux density $B_{z}(y)$, so the Kim model and the exponential model are respectively expressed as follows:

$$
\begin{gathered}
\frac{J_{c}\left(B_{z}(y)\right)}{J_{c 0}}=\frac{B_{0}}{B_{0}+\left|B_{z}(y)\right|}, \\
\frac{J_{c}\left(B_{z}(y)\right)}{J_{c 0}}=\exp \left(-\left|B_{z}(y)\right| / B_{0}\right),
\end{gathered}
$$

where $B_{0}$ is a constant field that characterizes the degree of field dependence. The body force (Lorentz force density) in the film is

$$
\boldsymbol{f}=\boldsymbol{J} \times \boldsymbol{B} \text {. }
$$

The magneto-elastic problem of thin superconducting strip for the two different critical-state models 
has been studied in our preceding paper [16]. In the field ascent stage, the body force remains zero in the central region and increases in magnitude along y-direction, but it is always negative, which means the film with crack is subjected to compressive stress. During the field descent branch, the body force has both negative value and positive value. Its peak value moves towards the middle of the film, and the peak value decreases as the maximum applied field decreases. Therefore, at the intermediate state of field reduction, the crack may be subjected to the largest tensile body force. Attention is limited to the crack problem of thin film in the field reduction. Fig. 2 shows the variations of body force for the three models with the applied field reduction. There is an important distinction between the three models. Thus, it's essential to take field-dependent $J_{c}(B)$ into account for studying the fracture behaviors of superconducting film. Because the thickness is very small compared with other two dimensions, this is a plane stress problem. Since the crack is parallel to the $x$ axis, the effect of body forces $f_{x}$ along the $x$ direction at infinity can be neglected, i.e. $f_{x}=f_{z}=0$. The body force along $y$ axis becomes $f_{y}(y)=J_{x}(y) B_{z}(y)$. Due to the symmetry of the problem, it is convenient to analyze one quarter part of the film by means of finite element method.

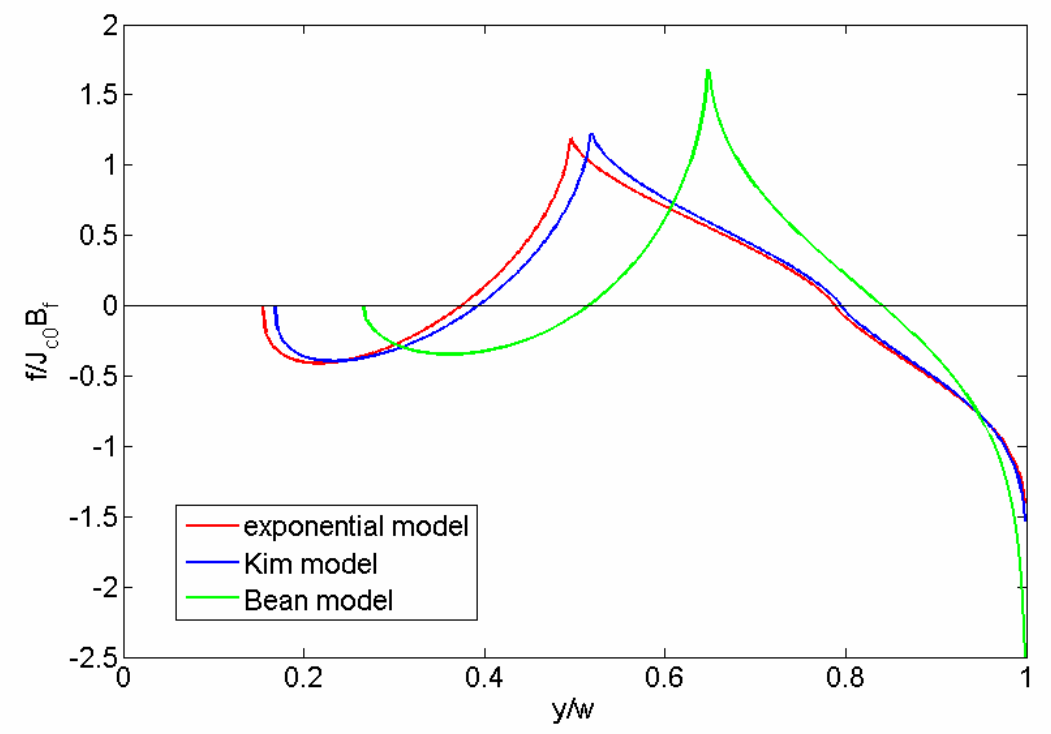

Figure 2. The variations of body force for the Bean model, Kim model and exponential model when the applied field decreases from the maximal value $2 B_{f}$ to 0 .

The stress intensity factor $K$ is an important parameter in fracture mechanics, which determines whether the crack propagates or not. The crack would start to propagate the stress intensity factor $K$ increases to the critical stress intensity factor $K_{c r}$. The critical stress intensity factor $K_{c r}$ is material parameters, which is independent of the mechanical load and the applied field. Since the body force is perpendicular to the crack, only the mode I crack is considered in this paper and the energy release rate $G_{I I}$ is neglected. For the linear elastic fracture mechanics, the energy release rate is defined by

$$
G=G_{I}=\lim _{\Delta a \rightarrow 0} \frac{1}{2 d \Delta a} \int_{0}^{\Delta a} \sigma_{y y}(x) \Delta v(x) d x,
$$

where $\Delta a$ is the increment of the crack length at the crack front. In addition, $\sigma_{y y}(x)$ and $\Delta v(x)$ are the normal stress and the virtual crack opening displacement at the extension of the crack, respectively. Because this equation is complex in calculation, Rybicki and Kanninen [21] proposed 
the famous virtual crack closure technique to calculate the energy release rate, which can save our computational time and has high precision with fewer meshes. Then the energy release rate can be obtained by

$$
G \cong \frac{F_{y} \Delta v}{2 d \Delta a},
$$

where $F_{y}, \Delta a$ and $\Delta v$ are the nodal force along $y$ direction at the crack tip, the width of the mesh and the crack opening displacement at the node near the crack tip, respectively. The stress intensity factor $K$, one of the most important parameters in linear elastic fracture mechanics, reflects the singularity of the stress at the crack tip. The relationship between the stress intensity factor $K$ and energy release rate $G$ is given by

$$
G=\frac{K^{2}}{E} \text {. }
$$

\section{Results and discussions}

In this section, we discuss the crack problems of thin superconducting film with one central crack. The stress intensity factor is normalized by

$$
K_{0}=\sigma_{0} \sqrt{a / \pi},
$$

where $\sigma_{0}=\mu_{0} J_{c 0}^{2} d w$. We mainly draw attention to the developments of stress intensity factor versus applied field and crack length for the three models.

Shown in Fig. 3 is the variation of $K / K_{0}$ for different critical state models when the applied field decreases from the initial value $2 B_{f}$ to 0 . It can be seen that the stress intensity factor firstly increases to the maximum value at a parameter field $B_{a}^{*}$, then decreases after it reaches peak value. Note that the superconducting film with crack is the most dangerous near the parameter field $B_{a}^{*}$ during the field descent. What's more, although the profiles of stress intensity factor between the exponential model and the Kim model is nearly the same, it is not difficult to find that the maximum stress intensity factor and the corresponding parameter field $B_{a}^{*}$ for the exponential model are larger than those for the Bean model and Kim model. These imply that the crack in the thin superconducting film will propagate more easily based on the results of the exponential model than that of the other two models. In addition, due to the trapped field, one can see obviously that the intensity factor cannot recover to zero even when the applied field decreases from $B_{a m}$ to zero. 


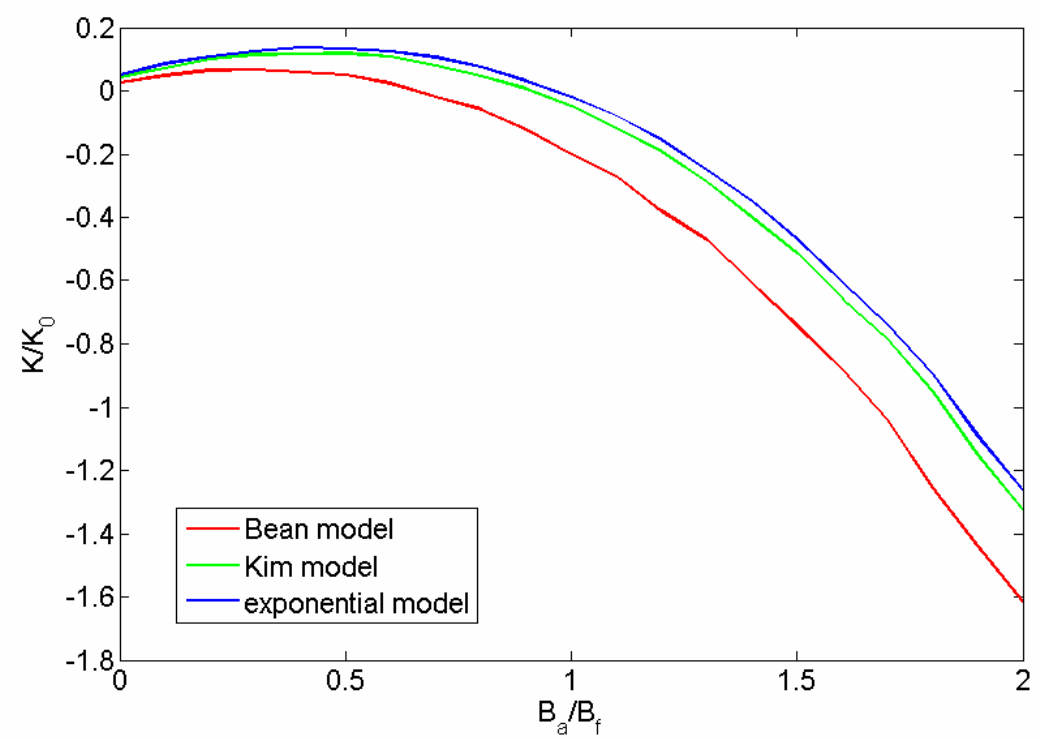

Figure 3. The stress intensity factors in the thin film with one central crack for the maximum field decreasing from $2 B_{f}$ to 0 .

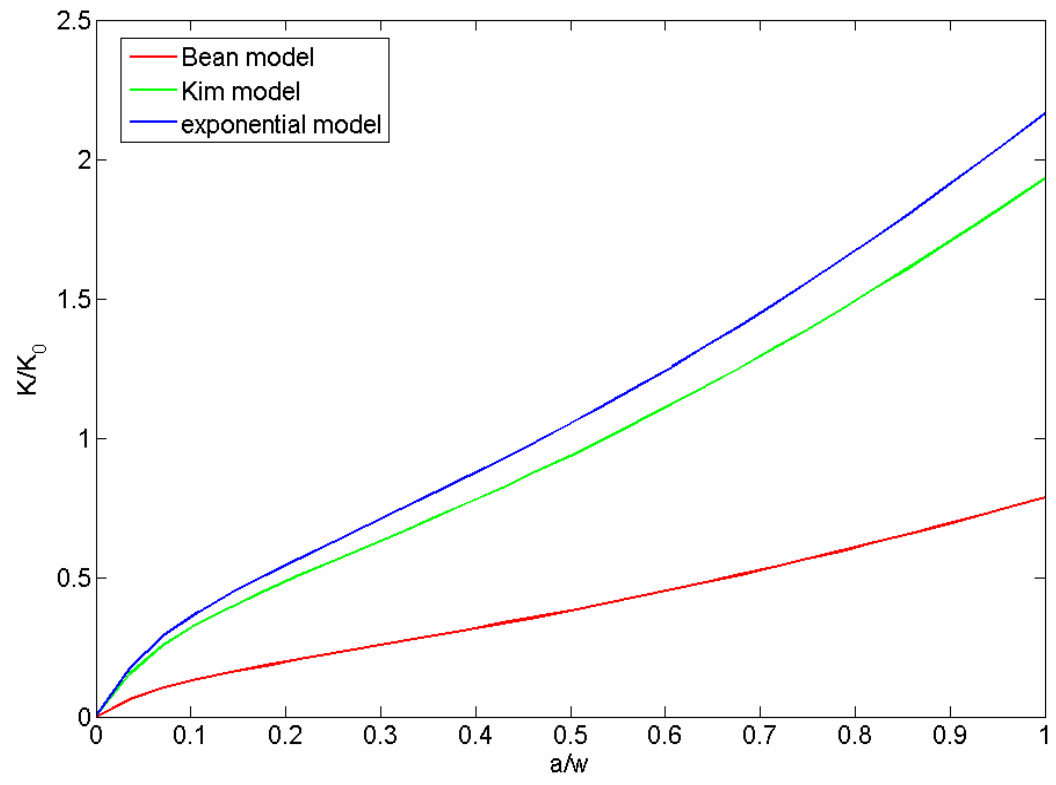

Figure 4. The stress intensity factors in the film with one central crack versus the crack length for the three models.

Fig. 4 shows the development of the stress intensity factor with respect to $a / w$ for different critical state models. As anticipated, the stress intensity factor $K$ increases as crack propagates, which indicates that the HTS film will have a tendency to break into pieces if the crack length increases to a critical value $a_{c r}$. One can tell the difference that the values derived from the exponential model increase more quickly than those from the other two models. That is to say, the effect of the crack length on stress intensity factor may be greater for the exponential model.

\section{Conclusions}

In this paper, we investigate the fracture behaviors induced by electromagnetic force with 
field-dependent critical current in thin superconducting film. Applying finite element method, the stress intensity factors of one central crack versus applied field and crack length are obtained for different critical state models. From the comparison between the three models, it can be found that the magnitudes of the $G$ and $K$ are remarkably different. Thus the prediction of parameter field $B_{a}^{*}$ corresponding to the overall maximum stress intensity factor, where the HTS thin film is the most dangerous, can be of practical importance. It is intended that these results presented in this paper may be useful for the interpretation of cracking and mechanical failure of HTS thin film in a number of real situations.

\section{Acknowledgements}

This research was supported by the Fund of Natural Science Foundation of China (Nos. 11032006, 11121202 and 11202087) and the Fundamental Research Funds for the Central Universities (lzujbky-2011-6) and Specialized Research Fund for the Doctoral Program of Higher Education under Grant 20110211120027. The authors gratefully acknowledge the support.

\section{References}

[1] S. Fleshler, L. T. Cronis, G. E. Conway, A. P. Malozemoff, T. Pe, J. McDonald, J. R. Clem, G. Vellego, P. Metra, Measurement of the ac power loss of $(\mathrm{Bi}, \mathrm{Pb})_{2} \mathrm{Sr}_{2} \mathrm{Ca}_{2} \mathrm{Cu}_{3} \mathrm{O}_{\mathrm{x}}$ composite tapes using the transport technique. Appl. Phys. Lett., 67 (1995) 3189.

[2] M. Suenaga, V. F. Solovyov, Q. Li, Z. Ye, H. J. Wiesmann, M. Iwakuma, M. Fukui, K. Toyota, F. Funaki, T. H. Johansen, D. V. Shantsev, J. R. Clem, AC losses in circular disks of thin $\mathrm{YBa}_{2} \mathrm{Cu}_{3} \mathrm{O}_{7}$ films in perpendicular magnetic fields. J. Appl. Phys., 94 (2003) 502.

[3] G. B. Lubkin, Applications of high-temperature superconductors spproach the marketplace. Phys. Today, 48 (1995) 20.

[4] A. Nabialek, H. Szymczak, V. A. Sirenko, A. I. D’yachenko, Influence of the real shape of a sample on the pinning induced magnetostriction. J. Appl. Phys., 84, 3770 (1998).

[5] T. H. Johansen, Flux-pinning-induced stress and strain in superconductors: Long rectangular slab, Phys. Rev. B, 59 (1999) 11187.

[6] T. H. Johansen, Flux-pinning-induced stress and strain in superconductors: Case of a long circular cylinder. Phys. Rev. B, 60 (1999) 9690.

[7] T. H. Johansen, Flux-pinning-induced stress and magnetostriction in bulk superconductors. Supercond. Sci. Technol., 13 (2000) R121.

[8] Y. Ren, R. Weinstein, J. Liu, R. P. Sawh, C. Foster, Damage caused by magnetic pressure at high trapped field in quasi-permanent magnets composed of melt-textured $\mathrm{Y}-\mathrm{Ba}-\mathrm{Cu}-\mathrm{O}$ superconductor. Physica C, 251 (1995) 15.

[9] P. Diko, Cracking in melt-processed RE-Ba-Cu-O superconductors. Supercond. Sci. Technol., 11 (1998) 68.

[10] P. Diko, G. Krabbes, Macro-cracking in melt-grown YBaCuO superconductor induced by surface oxygenation. Supercond. Sci. Technol., 16 (2003) 90.

[11] Y. H. Zhou, H. D. Yong, Crack problem for a long rectangular slab of superconductor under an electromagnetic force. Phys. Rev. B, 76 (2007) 094523.

[12] H. D. Yong, Y. H. Zhou, J. Zeng, Crack problem in a long cylindrical superconductor. J. Appl. Phys., 104 (2008) 113902.

[13] J. Zeng, Y. H. Zhou, H. D. Yong, Fracture behaviors induced by electromagnetic force in a long cylindrical superconductor. J. Appl. Phys., 108 (2010) 033901.

[14] H. D. Yong, Y. H. Zhou, Crack Problem for thin superconducting strip in a perpendicular magnetic field. IEEE Trans. Appl. Supercond., 22 (2012) 8400905. 
[15] H. D. Yong, Y. H. Zhou, Interface crack between superconducting film and substrate. J. Appl. Phys., 110 (2011) 063924.

[16] C. Xue, A. He, H. D. Yong, Y. H. Zhou, Magneto-elastic behaviour of thin type-II superconducting strip with field-dependent critical current. J. Appl. Phys., 2 (2013) 113.

[17] H. Ikuta, K. Kishio, and K. Kitazawa, Critical state models for flux-pinning-induced magnetostriction in type-II superconductors. J. Appl. Phys., 76 (1994)4776

[18] K. Yamafuji, T. Fujiyoshi, K. Toko, T. Matsuno, T. Kobayashi, and K. Kishio, On the magnetic field dependence of critical current density in single crystals of high- $\mathrm{T}_{\mathrm{C}}$ superconductors. Physica $\mathrm{C}$, 226 (1994) 133.

[19] S. Senoussi, M. Oussena, G. CoIlin, and I. A. Campbell, Exponential $H$ and $T$ decay of the critical current density in $\mathrm{YBa}_{2} \mathrm{Cu}_{3} \mathrm{O}_{7-\delta}$ single crystals. Phys. Rev. B, 37 (1998) 9792.

[20] J. McDonald, J. R. Clem, Theory of flux penetration into thin films with field-dependent critical current. Phys. Rev. B, 53 (1996) 8643.

[21] E. F. Rybicki, M. F. Kanninen, A finite element calculation of stress intensity factors by a modified crack closure integral. Eng. Fract. Mech., 9 (1977) 931. 